University of New Hampshire

University of New Hampshire Scholars' Repository

Physics Scholarship

Physics

5-15-1997

\title{
First energetic neutral atom images from Polar
}

M. G. Henderson

G. D. Reeves

Harlan E. Spence

Boston University, harlan.spence@unh.edu

R. B. Sheldon

A. M. Jorgensen

See next page for additional authors

Follow this and additional works at: https://scholars.unh.edu/physics_facpub

Part of the Physics Commons

\section{Recommended Citation}

Henderson, M. G.; Reeves, G. D.; Spence, Harlan E.; Sheldon, R. B.; Jorgensen, A. M.; Blake, J. B.; and Fennell, J. F., "First energetic neutral atom images from Polar" (1997). Geophysical Research Letters. 297. https://scholars.unh.edu/physics_facpub/297

This Article is brought to you for free and open access by the Physics at University of New Hampshire Scholars' Repository. It has been accepted for inclusion in Physics Scholarship by an authorized administrator of University of New Hampshire Scholars' Repository. For more information, please contact Scholarly.Communication@unh.edu. 


\section{Authors}

M. G. Henderson, G. D. Reeves, Harlan E. Spence, R. B. Sheldon, A. M. Jorgensen, J. B. Blake, and J. F. Fennell 


\title{
First energetic neutral atom images from Polar
}

\author{
M. G. Henderson ${ }^{1}$, G. D. Reeves ${ }^{1}$, H. E. Spence ${ }^{2}$, R. B. Sheldon ${ }^{2}$, A. M. Jorgensen ${ }^{2}$, \\ J. B. Blake ${ }^{3}$, and J. F. Fennell ${ }^{3}$
}

\begin{abstract}
Energetic neutral atoms are created when energetic magnetospheric ions undergo charge exchange with cold neutral atoms in the Earth's tenuous extended atmosphere (the geocorona). Since they are unaffected by the Earth's magnetic field, these energetic neutrals travel away in straight line trajectories from the points of charge exchange. The remote detection of these particles provides a powerful means through which the global distribution and properties of the geocorona and ring current can be inferred. Due to its $2 \times 9 R_{E}$ polar orbit, the Polar spacecraft provides an excellent platform from which to observe ENAs because it spends much of its time in the polar caps which are usually free from the contaminating energetic charged particles that make observations of ENAs more difficult. In this brief report, we present the first ENA imaging results from Polar. Storm-time ENA images are presented for a northern polar cap apogee pass on August 29, 1996 and for a southern polar cap perigee pass on October 23, 1996. As well, we show with a third event (July 31, 1996) that ENA emissions can also be detected in association with individual substorms.
\end{abstract}

\section{Introduction}

Energetic neutral atoms (ENAs) are produced when energetic magnetospheric ions undergo charge-exchange collisions with the thermal neutral atoms that make up the Earths extended atmosphere (the geocorona). This type of interaction can take place in the ring current, the mid and auroral latitude energetic particle precipitation zones, and within the low altitude equatorial ion belt [Hovestadt et al., 1972; Moritz, 1972] that is itself formed by ionization of earthward directed ENAs via collisions in the low altitude equatorial atmosphere. Once an energetic neutral atom is created, it moves away in a straight-line trajectory from the point of charge exchange and can therefore be detected remotely by an appropriately instrumented spacecraft. Since these neutrals are continuously being emitted from the charge exchange regions in all directions with energies and fluxes directly dependent upon the properties of the ions and geocorona, they carry important information on the global characteristics of both the geocorona and the magnetospheric ion population.

The study of ENAs in the Earth's magnetosphere has had a long history. The earliest evidence that energetic hydrogen exists in the near-earth space environment came from observations of hydrogen emission lines in auroral spectra made by Vegard [1939]. Later on, following the discovery of the radiation belts and ring current, and the dynamics associated with geomagnetic storms, many researchers came to the conclusion that ENA-producing charge exchange pro-

\footnotetext{
${ }^{1}$ Los Alamos National Laboratory, Los Alamos, NM.

${ }^{2}$ Center for Space Physics, Boston University, Boston, MA

${ }^{3}$ The Aerospace Corporation, Los Angeles, CA

Copyright 1997 by the American Geophysical Union.
}

Paper number 97GL01162.

0094-8534/97/97GL-01162\$05.00 cesses were an important mechanism for the decay of the storm-time ring current (e.g. see Roelof et al. [1985]). The first suggestion that ENAs emitted from the radiation belts and ring current could be used to remotely sense the magnetospheric energetic ion population was made by Hovestadt and Scholer, [1976], and the first global image of ENA emissions was produced by Roelof [1987] from data acquired by the ISEE1 spacecraft. Also, the first composition measurements of ENAs have been reported recently by Lui et al. [1996]. See Williams et al. [1992] for a more comprehensive and detailed review of ENA imaging (as well as other types of magnetospheric imaging).

In this brief report we present the first ENA imaging results from the Polar spacecraft.

\section{Instrumentation}

The data presented here were acquired with the Imaging Proton Spectrometer (IPS) which is part of the Comprehensive Energetic Particle and Pitch Angle Distribution (CEPPAD) experiment on Polar. The IPS measures protons with energies in the range $20-1500 \mathrm{keV}$ in 16 energy channels over 9 separate polar-angle look directions simultaneously. As shown in figure 1, the central look-directions for the 9 detectors are arranged to be at $10^{\circ}, 30^{\circ}, 50^{\circ}, 70^{\circ}$, $90^{\circ}, 110^{\circ}, 130^{\circ}, 150^{\circ}$, and $170^{\circ}$ with respect to the spin axis and each detector has a field of view of $20^{\circ}$ in the polar direction by $11.25^{\circ}$ in the azimuthal direction which gives IPS a combined instantaneous field of view of $180^{\circ} \times 11.25^{\circ}$. For the integral energy channels (which are used here) the counts are accumulated into 32 sectors per spin (16 for the $10^{\circ}$ and $170^{\circ}$ detectors). Note that because the detectors rotate through $11.25^{\circ}$ during the accumulation interval, the effective angular response in the azimuthal direction for each sector is actually wider than $11.25^{\circ}$. For a detailed description of the IPS instrument see Blake et al. [1995].

Since the IPS cannot distinguish between ions and neutrals, ENAs can only be reliably identified when the flux of charged particles is very low. Fortunately, due to its highly elliptical polar orbit, Polar spends much of its time in the polar caps where this condition is usually met.

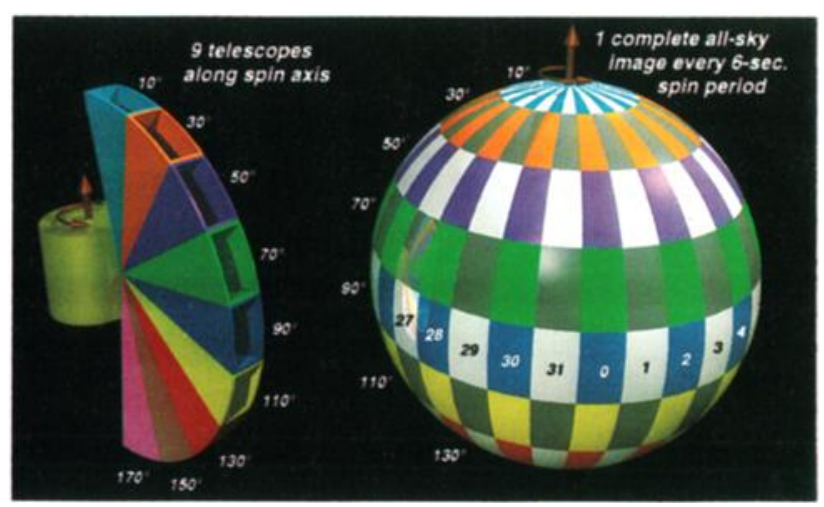

Figure 1. Schematic diagram illustrating how the nine IPS telescopes are oriented with respect to the spin axis. 


\section{Storm Time Energetic Neutral Atoms: August 29, 1996}
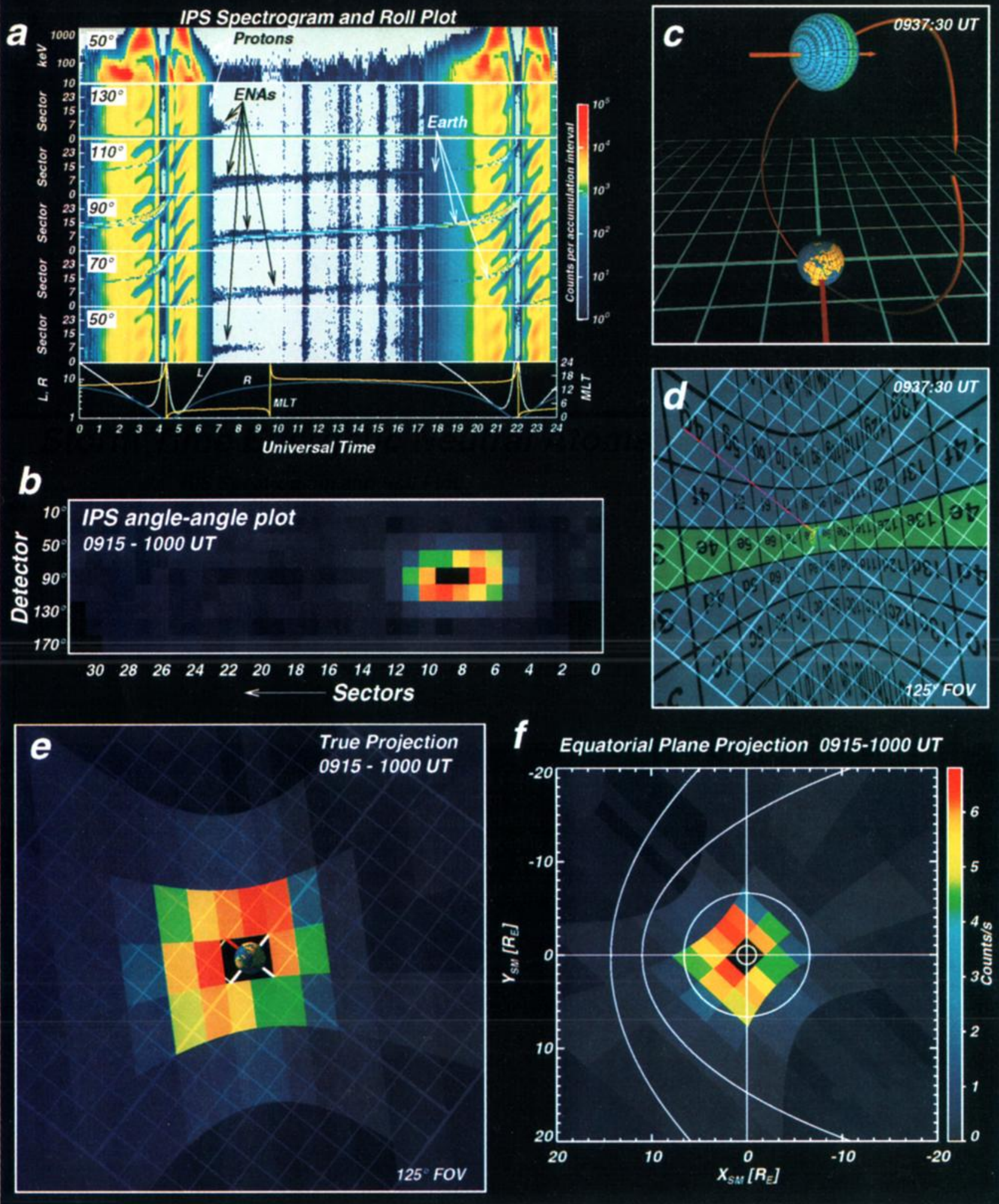

Figure 2. Storm-time ENA imaging on August 29, 1996. In (a), the IPS spectrogram from the $50^{\circ}$ detector is shown along with the (integral energy channel) sector vs. time plots from the $50^{\circ}, 70^{\circ}, 90^{\circ}, 110^{\circ}$, and $130^{\circ}$ detectors. An angle-angle plot (i.e. a polar-angle versus azimuthal-angle plot) constructed from this data is shown in (b). (c) shows the attitude and location of Polar in its orbit at 0937:30 UT in the solar magnetic coordinate system. The white mesh represents the SM $X-Y$ plane and the positive $\mathrm{x}$-axis is marked with a red line. The view that IPS (and each of the individual sectors) has of the Earth at 0937:30 UT is shown in (d). The final two panels show the resulting ENA image in a true projection (e) and in an equatorial SM plane projection (f). 


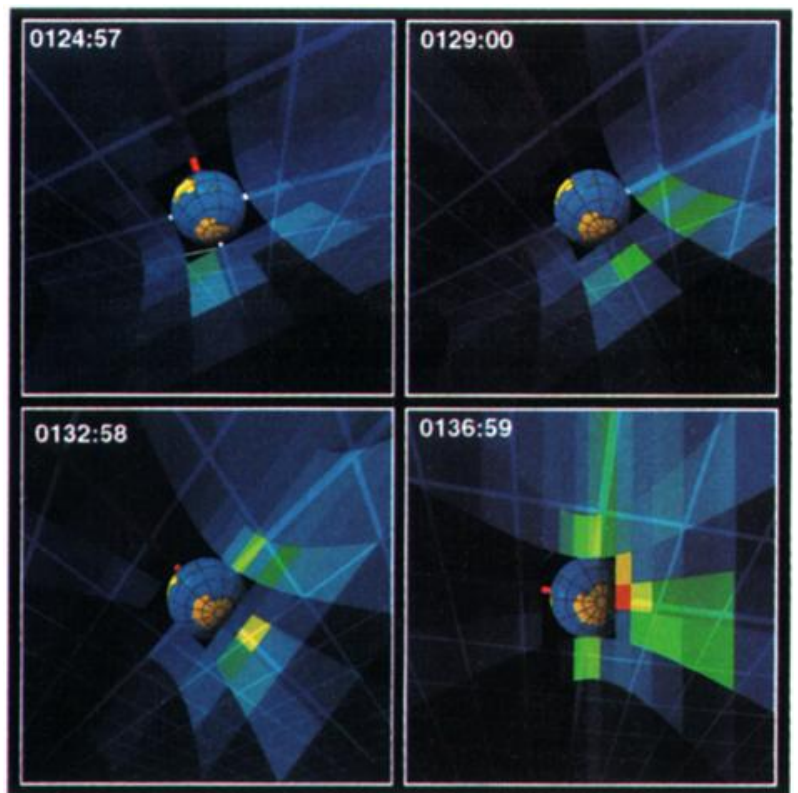

Figure 3. Storm-time ENA emissions during a southern polar cap pass on October 23, 1996.

\section{Observations}

\section{Storm-time ENAs: August 29, 1996}

In figure 2 we present observations of ENAs which were detected by the Polar CEPPAD/IPS instrument during a magnetic storm that occurred on August 29, 1996. The uppermost panel in figure 2a shows the (spin averaged) spectrogram from the $50^{\circ}$ detector while the next 5 panels show the (integral energy channel) sector vs. time plots from the $50^{\circ}-130^{\circ}$ detectors. In the lower-most panel, the radial distance (R), L-shell value (L), and magnetic local time (MLT) of Polar are also plotted as a function of time.

Since the orbital period of Polar is $\sim 18$ hours, the data presented in this 24-hour plot format spans more than one orbit. At the start of the day, Polar was situated $\sim 6 R_{E}$ above the SM $X-Y$ plane in the afternoon sector and was moving down toward perigee over the southern polar cap. The radiation belt/ring current ions were observed from 0000 to $\approx 0400$ UT and from $\approx 0420$ UT up until $\approx 0645$ UT. After this time, Polar entered the northern polar cap and remained there for many hours up until $1700 \mathrm{UT}$ at which time it re-entered the ring current/radiation belt regions on its way toward a second southern polar cap perigee pass.

Except for a series of relatively short-lived low-flux bursts of protons, the polar cap field lines were essentially devoid of significant charged particle fluxes. As mentioned earlier, this situation allows us to readily identify ENAs when they are present in sufficient numbers. In figure $2 a$, the enhanced storm-time ENAs can be seen as slightly inclined fuzzy horizontal bands in the northern polar cap (see arrows). Strong confirmation that these bands are indeed ENAs comes from the fact that the Polar CAMMICE/MICS instrument, which only measures charged particles (it rejects neutrals), did not see them. In addition to the ENA signatures, the response of IPS to Earth light - which is always observed regardless of whether or not ENAs are present - can be seen in the $90^{\circ}$ detector throughout the polar cap. This Earth response also shows up in the $70^{\circ}$ and $110^{\circ}$ detectors when Polar is nearer to the Earth (e.g. prior to $\sim 0900 \mathrm{UT}$ and after $\sim 1600 \mathrm{UT}$ ) due to the fact that the Earth fills more of the field of view at such times. Note that ENAs are only observed when the detectors are looking toward regions near Earth.
Figure 2b shows an angle-angle plot (polar-angle vs. azimuth angle) constructed by integrating the IPS integral energy channel counts over the time period 0915-1000 UT. This time span was chosen because it had a particularly low background of charged particle counts and because Polar was nearing apogee where the viewing geometry changes only very gradually with time. In this format, the directions from which the ENAs arrive in the spacecraft reference frame are most easily seen. The Earth response shows up in sectors 8 and 9 of the $90^{\circ}$ detector while the sun response shows up in sectors $0,1,30$, and 31 of the $130^{\circ}-170^{\circ}$ detectors. In order to enhance the visibility of the ENA fluxes, the Earth and Sun contaminated pixels have been blanked.

To additionally orient the reader, the location and attitude of Polar (at 0937:30 UT) is illustrated in figure 2c as it is approaching apogee. In this figure, the $2 \times 2 R_{E}$ white mesh represents the solar magnetic (SM) $X-Y$ plane where the positive $X$-axis is marked with a red line and the positive $Y$-axis is located in the lower right hand corner. The Earth is shown at the center of this mesh and the orbit and spin axis of Polar are shown as the copper-colored tubing. The blue and green sphere around Polar indicates where each of the sectors were pointing at 0937:30 UT.

Note that while the detectors make a complete revolution once per spin period, the sectors into which the counts are accumulated do not. The start of sector zero always occurs when the sun is (approximately) between sectors 31 and 0 , so the sector patterns do shift in time but only as a function of the orbital motion and not as a function of the spin phase. The view that IPS has of the Earth at 0937:30 UT is shown in figure 2d. Each sector is annotated with its sector number and a letter identifying the associated detector (' $a$ ' for the $10^{\circ} ;$ ' $i$ ' for the $170^{\circ}$ detector). As noted above, at 0937:30 UT the Earth lies in sectors 8 and 9 of the $90^{\circ}$ detector.

A true projection ENA image derived from the angle-angle plot shown in figure $2 b$ is shown in figure $2 e$ in the same format as figure 2d. As expected, due to the build-up of an enhanced storm-time ring current during this event, a ring of enhanced ENA emissions is observed to encircle the Earth out to radial distances near geosynchronous orbit. The same data is also presented in an equatorial SM plane projection in figure $2 \mathrm{f}$ with the bowshock, magnetopause, and geosyn-

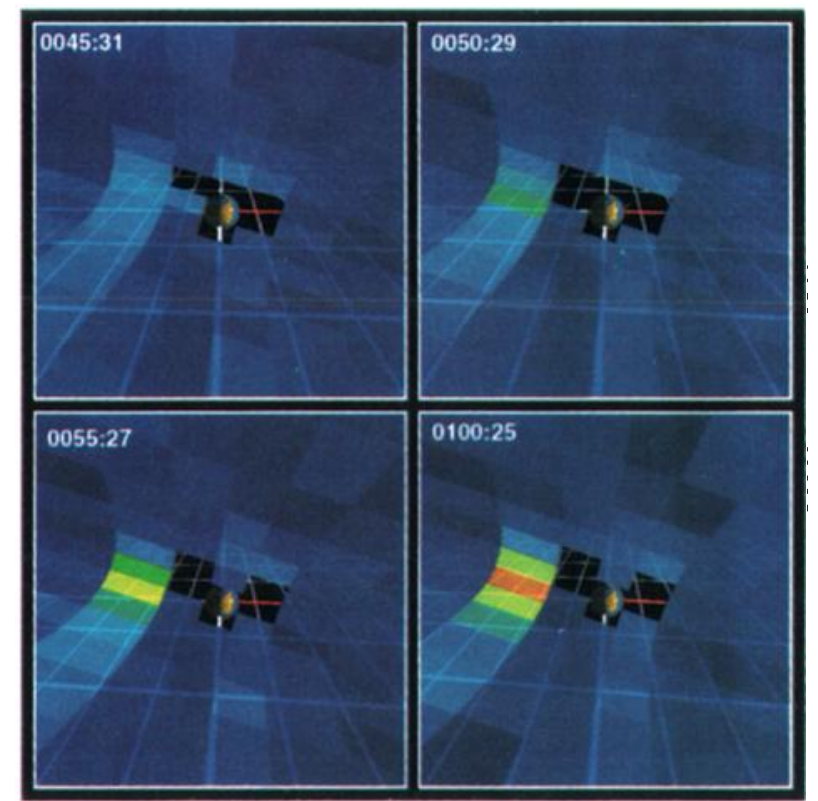

Figure 4. Substorm-associated ENA emissions on July 31, 1996. Polar is in the dawn sector and noon is to the right. 
chronous orbit shown for reference. The color bar associated with this plot also applies to the true projection image shown in figure $2 e$ and to the angle-angle plot shown in figure $2 b$.

\section{Storm-time ENAs: October 23, 1996}

In addition to the northern polar cap passes, ENAs can also be observed during the southern polar cap passes. In figure 3 we present a sequence of four southern pass ENA images acquired during a magnetic storm on October 23, 1996. Since Polar is so close to the Earth during this time period the viewing geometry changes rapidly with time. To avoid image blurring, the integration times are necessarily shorter. But this is compensated for by the fact that the counting rates are also much higher than they are at apogee. Note that equatorial plane projections are inappropriate for these kind of oblique viewing geometries. This complicates the interpretation of dynamics in such cases, but the many different projections that can be acquired over short periods of time also provide more information on the three dimensional structure of the charge exchange regions.

\section{Substorm-associated ENAs: July 31, 1996}

A new and unexpected result of the present study is that ENAs can also be observed in association with individual substorms. In figure 4 we show a sequence of ENA images acquired early on July 31, 1996 during a magnetospheric substorm. As shown, a significant brightening of the ENA emissions occurred on the night-side of the Earth between about 4 and $8 R_{E}$. Data from the Los Alamos Geosynchronous energetic particle detectors shows that this brightening occurred following a substorm-associated energetic particle injection. As well, the global auroral imagery from the Polar VIS instrument shows a very expanded nightside auroral distribution consistent with the occurrence of a magnetospheric substorm (L. Frank, pers. comm.).

From the observed fluxes of ENAs, we estimate with a very simple model the near-equatorial ion fluxes in the source region. A rigorous inversion of the ENA fluxes is beyond the scope of the present analysis; rather, we seek only an order-of-magnitude estimate of the average ion flux near $6 R_{E}$. First, we assume that the ENA emissions come from an "optically" thin medium. With this excellent approximation the differential ENA flux, is given by the lineof-sight (LOS) integration $j_{\text {ena }}(E)=\sigma \int n_{H}(\ell) j_{\text {ion }}(E, \ell) d \ell$, where $\sigma$ is the $\mathrm{H}-\mathrm{H}^{+}$charge-exchange cross section, $n_{H}$ is the neutral hydrogen density, $j_{i o n}$ is the ion differential flux, $E$ is the particle energy, and $\ell$ is the LOS path length (see Lui et al. [1996]). We assume that the ion fluxes producing the ENAs come from a slab with height, $\mathrm{L}=$ $4 \mathrm{R}_{E}$, centered about the magnetic equator. Within the slab we assume that the neutral hydrogen and proton densities are uniform. The LOS integration can then be simplified to solve for the ion flux, $j_{2 o n}(E)=j_{\text {ena }}(E)\left(\sigma n_{H} L\right)^{-1}$. Hodges' [1994] exospheric model is used to estimate the average geocoronal neutral density which, at a distance of 6 $\mathrm{R}_{E}$, yields a value of $\sim 100 \mathrm{~cm}^{-3}$. At onset, the brightest pixel (centered at $L=6$ ) recorded ENA differential fluxes of $\approx 3 \times 10^{2}\left(\mathrm{~cm}^{2} \cdot \mathrm{s} \cdot \mathrm{sr} \cdot \mathrm{keV}\right)^{-1}$ at $30 \mathrm{keV}$. At this energy, the $\mathrm{H}-\mathrm{H}^{+}$charge-exchange cross section is estimated to be $3 \times 10^{-16} \mathrm{~cm}^{2}$ Hodges [1994], so that the observed differential flux of $30 \mathrm{keV}$ ENAs corresponds to a $30 \mathrm{keV}$ ion differential flux of $\sim 4 \times 10^{6}\left(\mathrm{~cm}^{2} \cdot \mathrm{s} \cdot \mathrm{sr} \cdot \mathrm{keV}\right)^{-1}$ in the onset region. Differential fluxes comparable to and even considerably higher than this estimate are observed routinely by geostationary satellites during substorm injections. Despite the crudity of the model, this calculation provides initial confidence that the ENA intensity observed is consistent with the expected ion source population.

\section{Summary}

As demonstrated in this study, the IPS instrument on Polar can easily detect enhanced ENA emissions from the radiation belt/ring current regions with count rates sufficient for the construction of ENA images. In addition, Polar's $2 \times 9 R_{E}$ polar orbit allows us to monitor the ENA emissions continuously for many hours at a time. The images constructed for the August 29 and October 23 events show the expected enhancement of energetic neutrals arising from the growth and development of the storm-time ring current. And the images constructed for the July 31 event clearly show, for the first time, that ENAs can also be detected in response to individual substorm injections.

While an enormous amount of information can be obtained from the raw ENA images alone, it is important to note that they do not map out the distribution of the energetic ion population directly. Instead, they map out the regions of charge exchange collisions occurring between the energetic ion population and the Earth's geocorona. In future analysis, we plan to utilize forward modeling techniques (e.g. Roelof [1987]) in order to infer the true distribution of energetic ions.

As well, we plan to compare animated sequences of ENA images during substorms and during the development and decay of the storm-time ring current with global auroral imager data, in-situ particle measurements from other ISTP spacecraft, and ground-based observations. Spatial asymmetries and temporal and spectral variability in the ring current will be investigated. And we will attempt to quantify the importance of charge exchange processes in the decay of the ring current.

Acknowledgments. We gratefuly acknowledge the following key scientists and engineers who contributed significantly to the IPS instrument: S. Imamoto, B. Johnson, W. A. Kolasinski, D. Mabry, J. Osborn, J. Skinner, F. Hilsenrath, C. Wilbur. This work was supported under NASA grant number S19511E.

\section{References}

Blake, J. B., et al., CEPPAD experiment on POLAR, Space Sci. Rev., 71, 531, 1995.

Hodges, R. R., Monte Carlo simulation of the terrestrial hydrogen exosphere, J. Geophys. Res., 99, 23229, 1994.

Hovestadt, D., B. Häusler, and M. Scholer, Observation of energetic particles at very low altitudes near the geomagnetic equator, Phys. Rev. Lett, 28, 1340, 1972 .

Lui, A. T. Y., D. J. Williams, E. C. Roelof, R. W. McEntire, and D. G. Mitchell, First composition measurements of energetic neutrals, Geophys. Res. Lett., 23, 2641, 1996.

Moritz, J., Energetic protons at low equatorial altitudes, Z. Geophys., 38, 701, 1972.

Roelof, E. C., Energetic neutral atom image of a storm-time ring current, Geophys. Res. Lett., 14, 652, 1987.

Roelof, E. C., D. G. Mitchell, and D. J. Williams, Energetic neutral atoms $(E \approx 50 \mathrm{kev})$ from the ring current: IMP 7/8 and ISEE 1, J. Geophys. Res., 90, 10991, 1985.

Vegard, L., Hydrogen showers in the auroral region, nature, 144 , $1089,1939$.

Williams, D. J., E. C. Roelof, and D. G. Mitchell, Global magnetospheric imaging, Rev. Geophys., 30, 183, 1992.

M. G. Henderson, G. D. Reeves, Mail Stop D-436, Los

Alamos National Laboratory, Los Alamos, NM 87545, U.S.A.

H. E. Spence, R. B. Sheldon, and A. M. Jorgensen, Center for Space Physics, Boston University, Boston, Mass., U.S.A

J. B. Blake, and J. F. Fennell, The Aerospace Corporation, Los Angeles, CA 90009, U.S.A.

(Received: March 10, 1997; accepted: March 18, 1997.) 